\title{
Increase of Capacity in Electric Arc-Furnace Steel Mill Factories by Means of a Demand-Side Management Strategy and Ampacity Techniques
}

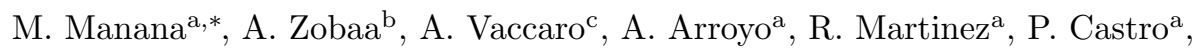 \\ A. Laso $^{\text {a }}$, S. Bustamante ${ }^{a}$ \\ ${ }^{a}$ University of Cantabria, Spain \\ ${ }^{b}$ Brunel University, UK \\ ${ }^{c}$ University of Sannio, Italy
}

\begin{abstract}
An electric arc-furnace is a complex industry which demands high levels of electrical energy in order to heat iron materials and other additives needed for the production of cast iron and/or steelmaking. The cost of the electrical energy demanded by the factory during the production can be greater than $20 \%$ of the overall cost. This kind of arc-furnace allows the production of steel with levels of scrap metal feedstock up to $100 \%$. From an electrical point of view, the factory size in terms of its maximum apparent power demanded from the grid is designed to make use of the static capacity of the transmission line that supplies the energy. In that case, it is not possible to increase the power of the factory above the static rating by adding new facilites without installing new transmission infrastructures. This paper presents a methodology that allows an increase in net power of an arc-furnace factory without installing new transmission lines. The solution is based on a mix strategy that combines Demand-Side Management (DSM) methodologies and the use of ampacity techniques according IEEE 738 and CIGRE TB601.

The application of DSM methodologies provides an improvement in the sustainability of not only the industrial customer but also in the overall grid. As a
\end{abstract}

\footnotetext{
* Corresponding author

Email address: mananam@unican.es (M. Manana)
}

Preprint submitted to International Journal of Electrical Power \& Energy SystemsMarch 7, 2020 
secondary effect, it reduces operational costs and the greenhouse gas emissions.

The proposed methodology has been tested in an arc-furnace factory located in the North of Spain.

Keywords: Demand-side management, electric arc-furnace, ampacity

\section{Introduction}

An electric arc furnace (EAF) is a furnace that heats charged material by using an electric arc. They are usually devoted to the production of cast iron and/or steelmaking. From an energy point of view, it is a load that it is intensive 5 in terms of its power demanded from the grid [1].

As the electrical energy cosumption in an EAF based steel factory is typically greater than $20 \%$ of the overall production cost, most of the modelling effort has been devoted to the analysis of energy comsuption considering cost minimization 2] as the main target [3].

On the other hand, Demand Side Management (DSM) can be considered as a set of measures defined in order to improve the way in which energy is demanded at the consumption side [4. Although DSM has traditionally been applied to residential loads, it has also been used in industrial environments [5].

In [6], the potential of DSM methodologies was investigated in applications specifically devoted to power-intensive industries. The main conclusion in the case of EAF is that DSM potential is strictly devoted to load shedding. The authors of 7 introduced an optimization method that can be applied to both batch and continuous-type loads.

DSM doesn't provide a single solution, but an action plan that includes several approaches: i) the improvement of energy efficiency by upgrading load technologies, ii) the reduction of energy cost by improving energy tariffs with incentives for specific consumption patterns [8, 9] and iii) the application of complex real-time control methodologies of distributed energy resources. In Palensky et al. [10] DSM is categorized into the following list: Energy Efficiency ${ }_{25}$ (EE), Time of Use (TOU), Demand Response (DR) and Spinning Reserve (SR). 
Fig. 1 summarizes the basic architecture and components of a DSM system that includes elements such as local generators, smart devices, energy storage systems, sensors and energy management units (EMU) [11. All the elements must to be connected through a communication infrastructure. An external connection via Internet can be provided depending on security issues. The application of DSM can change the operational conditions of the involved elements, forcing the network operator to adjust the capacity ratings $[12$.

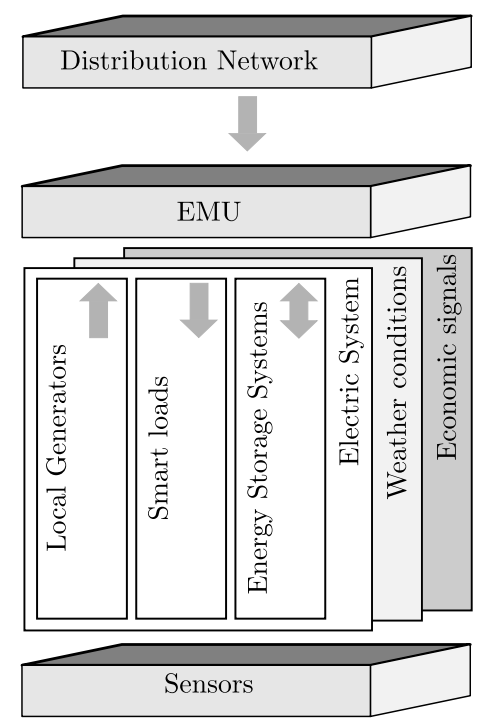

Figure 1: Architecture of a DSM system.

Load control algorithms have to deal with two main tasks:

1. Control the overall factory maintaining operational criteria.

2. Control each individual load to ensure that the total current demanded is below the dynamic rate. The individual control of loads can be on/off or proportional depending on the load.

Despite the fact that DSM techniques are well known, it is difficult to find references in which this methodology is applied to EAF applications [13, 14]

40 [15] 16] [17. Dalle et al. 18, analyzed how DSM can improve the electrode degradation in EAF. 
This paper proposes a non-classical application of DSM strategies in which the function to optimize is not economic cost, but the maximum current through the supply infrastructure considering that some loads inside the factory are dispatchables.

In this paper, we propose a methodology that combines both DSM techniques and dynamic line rating in order to increase the power capacity of a large electric arc-furnace factory without upgrading the overhead transmission line that links the factory to the transmission grid. This approach is especially useful in cases in which the factory is near urban areas where the constraints related to the construction of new transmission infrastructures are critical. The paper illustrates the proposed methodology for a real case in which an electric arc-furnace factory needs to increase its power demands, but the overhead infrastructure that supplies energy is already operating following a classical approach near the static limit. In the real case under study, the only available solution is to install a new transmission line or repower the existing one. In this research work, DSM combined with ampacity techniques is shown to increase the capacity of existing factories. The rest of this paper is organized as follows: Section 2 gives a general introduction to the way in which electric arc-furnaces demand energy. In Section 3 a demand-side management approach devoted to this type of factories is defined. In Section 4 we introduce the ampacity formulation that allows one to extend the capacity of the line according the weather conditions. In Sections 5 and 6 we introduce the concept of Quality of Service, that defines the impact of DSM and the optimization problem. In Section 7 the proposed methodology is applied to a real case considering a year of sampled data. Finally, the paper summarizes our conclusions and remarks on future directions.

\section{Electric arc-furnace energy demand}

A steel factory is a complex system that integrates two different sets of loads:

- High-energy demand loads with poor power quality requirements such as 
the electric arc-furnace.

- Medium and low-energy demand loads with restrictive power quality preliminary conditions (e.g., electronic controllers, power electronic devices, electrical drives, sensitive instrumentation).

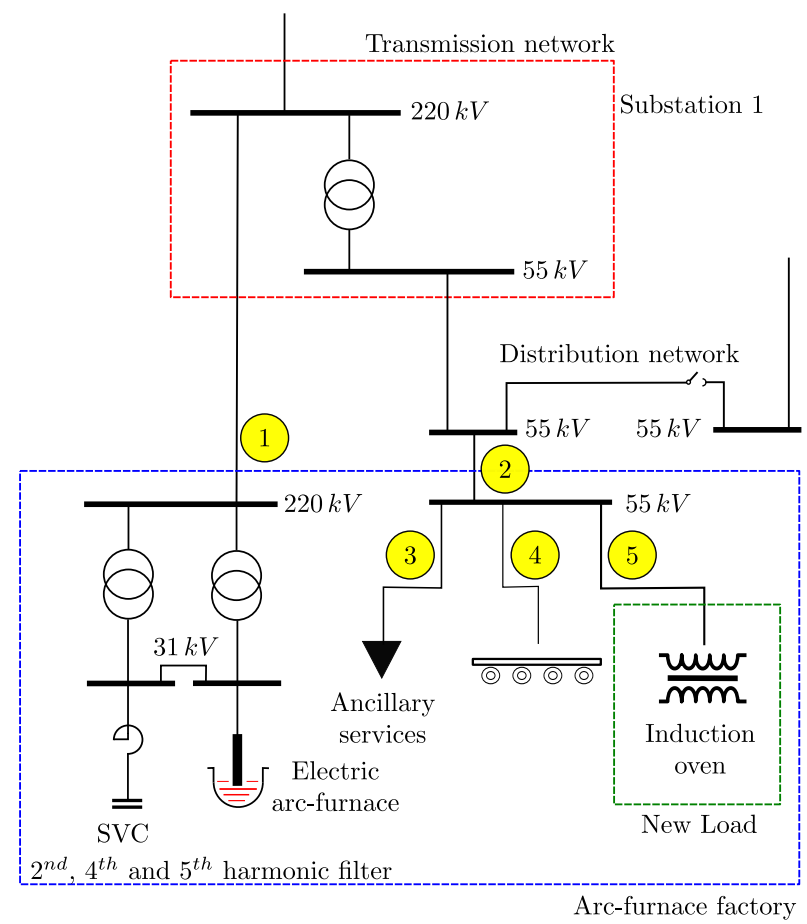

Figure 2: Simplified electric diagram of an electric arc-furnace factory with measurement points.

Fig. 2 shows the basic architecture of the electric-arc furnace factory. The industry is supplied by two overhead lines at $220 \mathrm{kV}$ (1) and $55 \mathrm{kV}$ (2). The $220 \mathrm{kV}$ line supplies energy to the electric arc-furnace used for steelmaking. This consumption is isolated from the rest of loads due to the high level of disturbances produced. The $55 \mathrm{kV}$ line supplies energy with better power quality levels to several sets of loads:

- Auxiliary services (3). All the electrical consumption of the treatment 
plant, including blowers, air filters, air compressors and other auxiliary elements.

- Hot-rolling mill (4). The steel billets manufactured by the electric arcfurnace are processed by circulating through several sets of pairs of rollers to reduce the thickness and making the thickness uniform.

The current rate of both lines has been defined considering a conservative approach. In the case of the $55 \mathrm{kV}$ line, the current rate is $560 \mathrm{~A}$. Considering a nominal voltage of $U_{N}=55 \mathrm{kV}$, the maximum capacity of this line is $S_{N}=$ 53.34 MVA. The aggregated consumption of this line is summarized in the histogram shown in fig. 3.

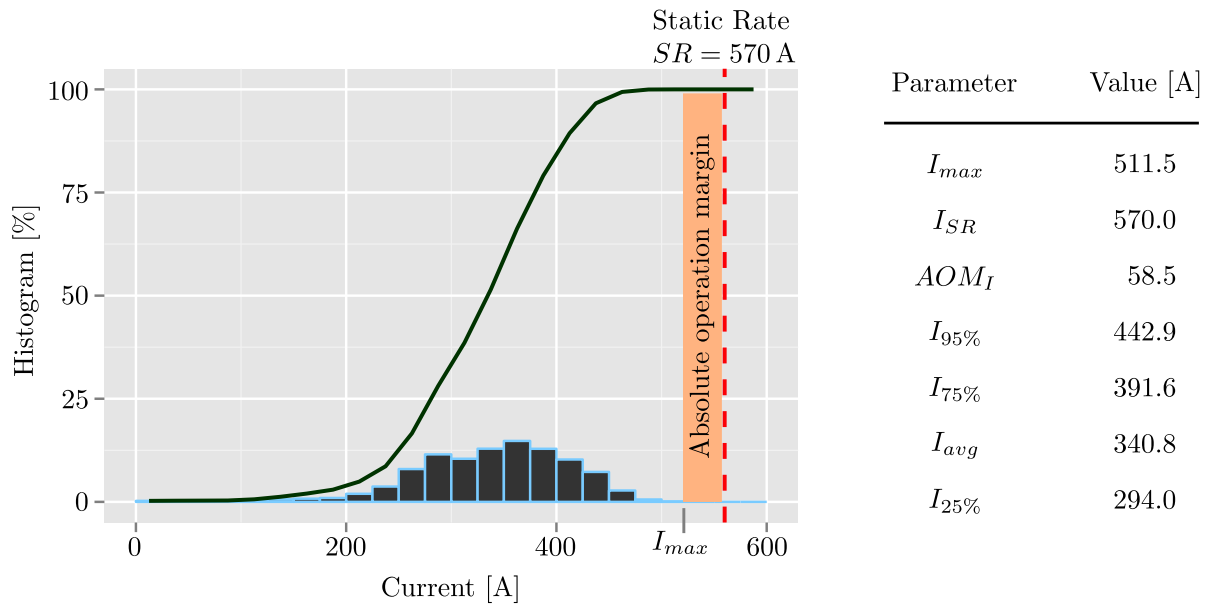

Figure 3: Histogram of the current demand at $55 \mathrm{kV}$ line.

A parameter defined as $A O M$ (Absolute Operation Margin) is defined in order to measure the available capacity of the transmission line that supplies energy to the steel factory. This parameter can be specified in terms of current or power.

The orange area in fig. 3 is the Absolute Operation Margin of the current $\left(A O M_{I}\right)$ defined as the difference between the static rate of the line $I_{S R}$ that 
supplies energy to the factory and the maximum value of current $I_{\max }$ demanded by the factory.

$$
A O M_{I}=I_{S R}-I_{\max }
$$

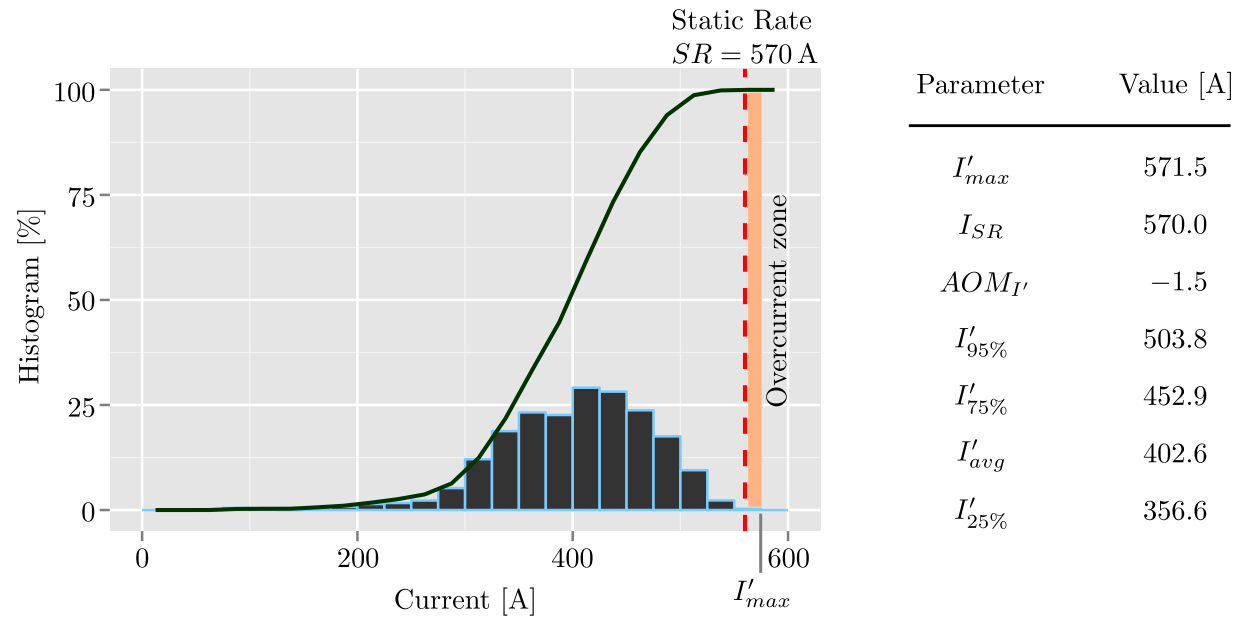

Figure 4: Histogram of the new demand at $55 \mathrm{kV}$ line.

Fig. 4 shows that the increase in the power demanded by the factory moves the value of $A O M_{I}$ from a positive to a negative one, $A O M_{I^{\prime}}$. In this new operating condition, the maximum current $I_{\max }^{\prime}>I_{S R}$ is inside the overcurrent zone, and thus the overhead line protection will be trigged.

The operational margin $A O M$ can be incremented in two main ways: 
1. By reducing the maximum value of the demanded apparent power $S_{\max }$. Demand-side management techniques can be used to control the overall set of loads inside the factory [19].

2. By increasing the transmission line static rate $S_{S R}$. Ampacity techniques can increase the dynamic capacity of transmission lines without the need to upgrade the infrastructure.

Both techniques are reviewed in the next sections.

\section{Demand-Side Management Techniques}

From a general point of view, all the electric loads that can be found in the factory can be classified in three main sets according to the way in which they are controlled, the constrains defined by the manufacturing process, and the quality of service [11:

\section{Fixed.}

2. Shiftable.

3. Elastic.

The classical approach followed by the company in the past for the billet pre-heating, was to perform this task with a natural gas oven. This strategy is not as efficient as the equivalent one based on induction, because of the way in which the billet is heated. Induction heating is more efficient and also more flexible since the billet is heated in a more homogeneus way, and the system can be switched on and off quickly.

Considering that time is sampled in slots, then for each time slot $n \in \mathcal{N}$, the total active power $p_{n}^{T O T}$ can be computed as follows

$$
p_{n}^{T O T}=\sum_{f \in \mathcal{F}} p_{f n}^{\mathcal{F}}+\sum_{s \in \mathcal{S}} p_{s n}^{\mathcal{S}}+\sum_{e \in \mathcal{E}} p_{e n}^{\mathcal{E}} \forall n \in \mathcal{N}
$$
sumption of shiftable loads and $p_{e n}^{\mathcal{E}}$ is the power demanded by elastic loads. 


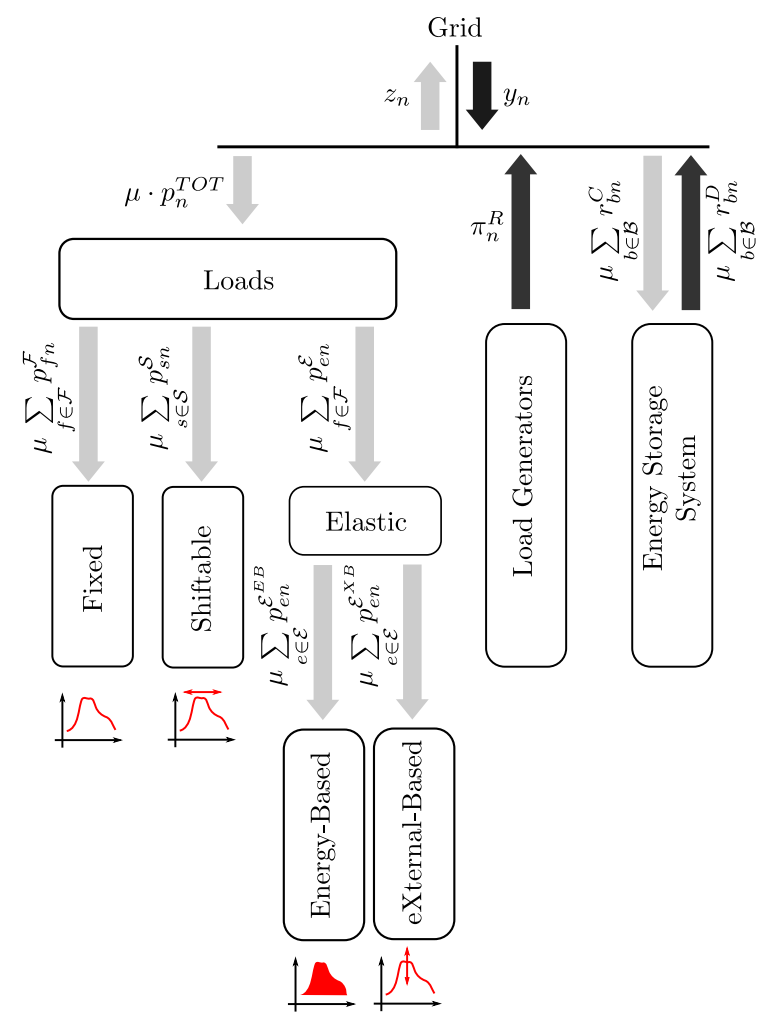

Figure 5: Power flows in the factory.

\subsection{Fixed loads}

A fixed load $\mathcal{F}$ is defined as a device or set of devices that cannot be controlled by the end-users. Both power consumption and operational time have fixed values (e.g., devices devoted to security issues, lights). Each device with a fixed active power $f \in \mathcal{F}$ is characterized by its active power $p_{f n}^{\mathcal{F}}$, considering all the time slots of the period that is analized $n \in \mathcal{N}$.

\subsection{Shiftable loads}

A shiftable load is a device or set of devices with an active power consumption that has a fixed profile where its starting time can be controlled in a certain range. These loads are represented by $\mathcal{S}$, and each device $s \in \mathcal{S}$ has a length of $D_{s}^{\mathcal{S}}$ slots. The power profile $l_{s d}^{\mathcal{S}}$ of a device $s$ in the $d$-th time slot of each 
individual profile (with $d \in D_{s}^{\mathcal{S}}=\left\{1,2, \ldots, D_{s}^{\mathcal{S}}\right\}$ ) can be considered as constant. The main task of the DSM during the operation of shiftable loads is to define, for each device $s \in \mathcal{S}$, the sample at which the device starts to run. The operating condition will continue during a set of consecutive time slots $\mathcal{N}_{s}^{\mathcal{S}} \subseteq \mathcal{N}$. The set $\mathcal{N}_{s}^{\mathcal{S}}$ must be in a range defined by a minimum starting-time slot $S T_{s}^{\mathcal{S}}$ and a maximum end-time slot $E T_{s}^{\mathcal{S}}$ defined by the production schedule.

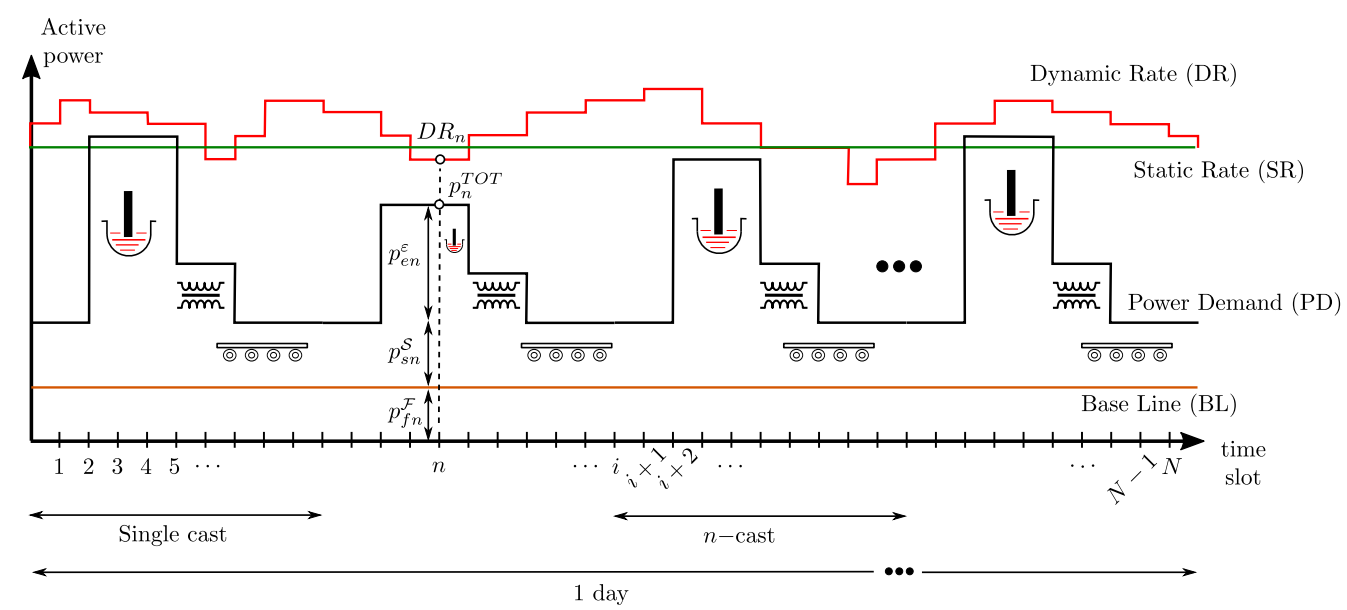

Figure 6: Profile of the active power demanded by the factory.

The scheduling decision problem of each shiftable device $s \in \mathcal{S}$ is defined by a binary variable $x_{s n}^{\mathcal{S}}$ for each sample $n \in \mathcal{N}$. The binary variable $x_{s n}^{\mathcal{S}}$ is one if the load $s$ starts in the sample $n$ and zero otherwise so $x_{s n}^{\mathcal{S}} \in\{0,1\}$. In addition, it is necessary to define a constraint that makes compulsory the operation of $s$ one time within the time slot $\mathcal{N}_{s}^{\mathcal{S}}[20,21]$.

$$
\begin{cases}\sum_{n=S T_{s}^{\mathcal{S}}}^{E T_{s}^{\mathcal{S}}-D^{\mathcal{S}}+1} x_{s n}^{\mathcal{S}}=1 & \forall s \in \mathcal{S} \\ x_{s n}^{\mathcal{S}}=0 & \forall s \in \mathcal{S}, n \in \mathcal{N} \backslash \mathcal{N}_{\mathcal{S}}^{\mathcal{S}}\end{cases}
$$

According to equation 3 the power $p_{s n}^{\mathcal{S}}$ demanded by each shiftable load 
$s \in \mathcal{S}$ in each time slot $n \in \mathcal{N}$ can be computed as

$$
p_{s n}^{\mathcal{S}}=\sum_{d \in D_{\mathcal{S}}^{\mathcal{S}}: d \leq n} l_{s d}^{\mathcal{S}} \cdot x_{s(n-d+1)}^{\mathcal{S}} \forall s \in \mathcal{S}, n \in \mathcal{N}
$$

\subsection{Elastic loads}

An elastic load is a device or set of devices, $\mathcal{E}$, whose instantaneous power demand can be managed by the DSM controller. Each device $e \in \mathcal{E}$ is represented by $p_{e n}^{\mathcal{E}}$ in each time slot $n \in \mathcal{N}$. There are two main types of elastic loads $e \in \mathcal{E}[22,23]$

- Energy-based elastic loads $\left(e \in \mathcal{E}^{E B}, \mathcal{E}^{E B} \subseteq \mathcal{E}\right)$.

- External-based elastic loads $\left(e \in \mathcal{E}^{X B}, \mathcal{E}^{X B} \subseteq \mathcal{E}\right)$.

The elastic load fulfills the condition

$$
\mathcal{E}=\mathcal{E}^{E B} \cup \mathcal{E}^{X B}
$$

\subsubsection{Energy-based elastic loads}

Energy-based elastic loads are sets of devices with a prescribed energy requirement. They are defined by a subset $\mathcal{E}^{E B} \subseteq \mathcal{E}$. All the appliances that belong to this group $e \in \mathcal{E}^{E B}$ demand a total amount of energy $E_{e, T O T}^{\mathcal{E}^{E B}}$ in the range of time slots $\mathcal{N}_{e}^{\mathcal{E}^{E B}} \subseteq \mathcal{N}$ defined by the range of time slots $\left[S T_{e}^{\mathcal{E}^{E B}}, E T_{e}^{\mathcal{E}^{E B}}\right]$. The energy $E_{e, T O T}^{\mathcal{E}^{E B}}$ can be computed as

$$
E_{e, T O T}^{\mathcal{E}^{E B}}=\mu \sum_{n=S T_{e}^{\mathcal{E}^{E B}}}^{E T_{e}^{\mathcal{E}^{E B}}} p_{e n}^{\mathcal{E}^{E B}} \forall e \in \mathcal{E}^{E B}
$$

where $\mu$ is a parameter equivalent to the time that depends on the duration of time slot $\Delta T_{n}$ with $\mu \leq \Delta T_{n}$. 


\subsubsection{External-based elastic loads}

Weather-based elastic loads are sets of devices which power demand depends on an external parameter. This external dependency is mainly based on weather conditions. They are defined by a subset $\mathcal{E}^{X B} \subseteq \mathcal{E}$. The external-based power $p_{\text {en }}^{\mathcal{E}^{X B}}$ can be represented as

$$
p_{e n}^{\mathcal{E}^{X B}}=\xi_{e n}^{\mathcal{E}^{X B}} \cdot p_{\text {en }}^{\mathcal{E}^{X, N O M}} \forall n \in \mathcal{N}
$$

where $\xi_{e n}^{\mathcal{E}^{X B}} \leq 1$ is the external conversion function representing the external parameter or set of parameters which modulate for each $n \in \mathcal{N}$ the power demanded by each load $e \in \mathcal{E}^{X B}$.

\subsection{Local Energy Generators}

Some industries have generation systems based on both renewable energies and classical internal combustion engines supplied by gas and fuel. In the case of renewable energy, the total amount of energy supplied, $\pi_{n}^{\mathcal{R}}$, is only a prediction for each sample $n \in \mathcal{N}$. Weather forecast is one of the most useful tools available for this task. It is assumed that energy produced is only for local consumption. If the energy produced is greater than the demand, then the difference $z_{n}$ will be injected into the grid for each $n \in \mathcal{N}$. The difference $z_{n}$ is equal or small that the net value $z_{n}^{\text {net }}$, where

$$
\pi_{n}^{n e t}=\max \left[0, \pi_{n}^{\mathcal{R}}-\mu \cdot p_{n}^{T O T}\right] \forall n \in \mathcal{N}
$$

\subsection{Energy Storage Systems}

Energy storage systems (ESS) provides a flexible tool to the DSM controller in order to modulate the energy consumption. The ESS can be in one of two states of the set $\mathcal{B}$ : i) charging $\left(\omega_{b n}\right)$ and ii) discharging $\left(1-\omega_{b n}\right)$. It is considered that a slot $n$ in which there is no charge or discharge can be considered a discharging state at near-zero discharging rate. The charging rate is defined by $200 r_{b n}^{C}$ while discharging rate is represented by $r_{b n}^{D}$. 


\subsection{Energy Balancing}

Once we know all the elements involved in the energy flux it is possible to define the balance condition for all the values of $n \in \mathcal{N}$ according the power flow summarized in Fig. 5 .

$$
y_{n}+\pi_{n}^{\mathcal{R}}+\mu \sum_{b \in \mathcal{B}} r_{b n}^{D}=z_{n}+\mu \sum_{b \in \mathcal{B}} r_{b n}^{C}+\mu \cdot p_{n}^{T O T}
$$

mal state of the conductor including the ambient temperature, solar radiation, and wind velocity. However, both methods represent different methodologies 
of calculation of the heat balance equation. The CIGRE model stablishes the steady-state heat balance equation that is shown in Eq. 10

$$
P_{J}+P_{M}+P_{S}+P_{I}=P_{C}+P_{R}+P_{W}
$$

230 heating, $P_{I}$ the corona heating, $P_{C}$ the convective cooling, $P_{R}$ the radiative cooling, and $P_{W}$ the evaporative cooling.

On the other hand the heat balance equation according to the IEEE standard doesn't consider magnetic heating, corona heating and evaporative cooling because their impact is usually insignificant compared with the other terms.

The simplified IEEE equation for non-steady-state heat balance includes the total heat capacity of the conductor $m C_{p}$, as shown below

$$
P_{J}+P_{S}+P_{I}=P_{C}+P_{R}+m C_{p} \frac{d T_{c}}{d t}
$$

In addition, the IEEE equation for steady-state heat balance neglects magnetic, corona and evaporative heat losses. In steady-state conditions $\frac{d T_{c}}{d t}=0$, so the current rating $I_{D R}$ can be computed as

$$
I_{D R}=\sqrt{\frac{P_{C}+P_{R}-P_{J}-P_{S}}{R\left(T_{c, a v g}\right)}}
$$

The overhead line $O_{1}$ (see Fig. 2) is operated following a static approach in which the capacity is defined in steady-state considering an ambient temperature of $40^{\circ} \mathrm{C}$ and a wind speed of $0,6 \mathrm{~m} / \mathrm{s}$.

If the line is operated in dynamic mode, then the increment of capacity $\Delta I_{D R}=I_{D R}-I_{S R}$ depends on the weather conditions. The values obtained acording eq. 12 are theoretical values that have to be limited taking into account the maximum operational limits of underground cable sections, breakers, current transormers, and all the elements connected in series with the overhead cable.

The transmission capacity of the line $S_{D R}$ can be computed as

$$
S_{D R}=\sqrt{3} V_{L L} I_{D R}
$$




\section{Quality of Service}

The Quality of Service $Q S$ is a parameter that refers to the ability of the Energy Control System (ECS) to fulfills all the requirements defined by the set of loads. The total value of the system $Q S^{T O T}$ reaches its maximum value when there are no constraints limiting the power demanded by all the loads included in the factory. If the ECS introduces any constrains in the behavior of the loads and the rest of the subsystems (generators and storage devices), then the $Q S_{n}^{T O T}$ at any time $n$ will be defined by eq. 14

$$
Q S_{n}^{T O T}=\sum_{f \in \mathcal{F}} Q S_{f n}^{\mathcal{F}}+\sum_{s \in \mathcal{S}} Q S_{s n}^{\mathcal{S}}+\sum_{e \in \mathcal{E}} Q S_{e n}^{\mathcal{E}} \forall n \in \mathcal{N}
$$

QS is used in order to define a weight for each load as not all the loads play a role with the same importance in the industrial process.

\section{Optimization}

Considering a power factor almost equal to 1.0, the active power is numerically identical to the apparent power so $P_{D R} \equiv S_{D R}$. Even in a case in which the $\mathrm{PF}$ is not equal to one, it is still possible to extend the formulation of the problem by applying the Boucherot's theorem to compute both the active and reactive power. After that, the apparent power can be translated to rms current directly. For any time slot $n$ the dynamic power capacity $p_{n}^{D R}$ defines the upper limit of the power that can be handled by the infrastructure. The mixed constrained minimization problem may be written as

$$
\begin{cases}\underset{\forall n \in \mathcal{N}}{\operatorname{minimize}} & \max \left[\left|y_{n}-z_{n}\right|\right] \\ \text { subject to } & \left|y_{n}-z_{n}\right| \leq \xi \cdot P_{n}^{D R}\end{cases}
$$

where $\xi$ is the security margin. The optimization process is summarized in algorithm 1. In the proposed methodology the optimization is performed using MonteCarlo simulation. It is assumed that MonteCarlo provides a near-optimal 
solution by sampling variables according specific probability distribution functions. This approach can be useful when the optimization problem has several local optima, the constraints are difficult to define, and the number or type of parameters is high 28. In spite of the fact that the solution is near-optimal, the computational burden of this method is low enough to be applied in real-time applications. Shabani et al. 29] propose a probabilistic methodology that can be applied to the computation of power cable ampacity by considering uncertainty of parameters and economic constraints in isolated conductors.

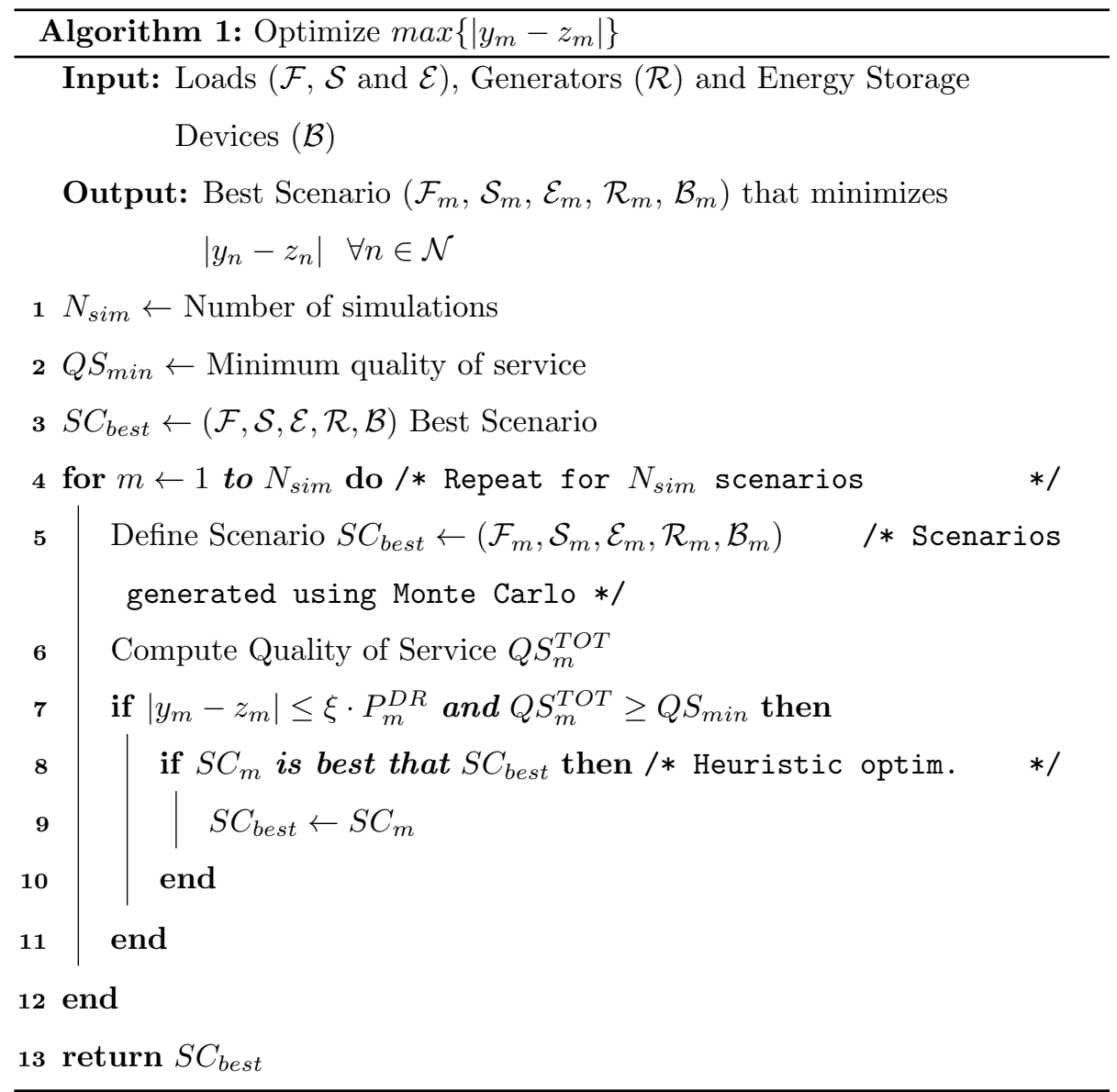




\section{Results}

The proposed methodology has been applied to the electric arc-furnace factory described in section 2. The analysis has been carried out measuring weather conditions over the course of one year. Fig. 7 summarizes the values of ampacity $\Delta I_{D R}$ during one year of measurements considering several degrees of aggregation (season, ambient temperature, and solar radiation).

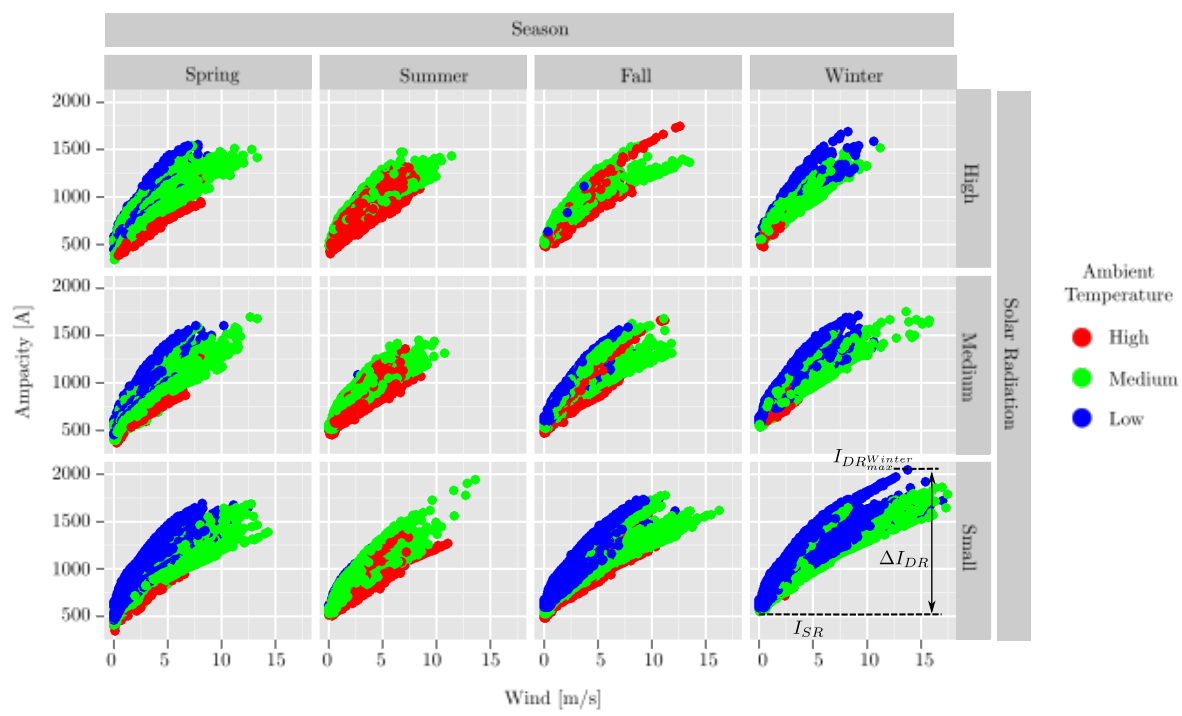

Figure 7: Dynamic capacity of the transmission line aggregated by season, solar radiation and ambient temperature.

Fig. 7 shows that the ampacity is greater that the static rating $I_{S R}$ and the maximum demand $I_{\max }^{\prime}$ most of the time. The aggregated data during a year is summarized in Fig. 8. a.

Fig. 8.b shows the period during which the DTR $I_{D R}$ is smaller than the required maximum current $I_{m a x}^{\prime}$, and where $\Delta t_{D S M}$ is equal to 650 hours a year, considering that the current demanded by the factory is $I_{\max }^{\prime}$ all the time. In a real case the current is fluctuating below that value so the proposed methodology for DSM will only have to be applied during a small percentage of this period $\Delta t_{D S M}$. 


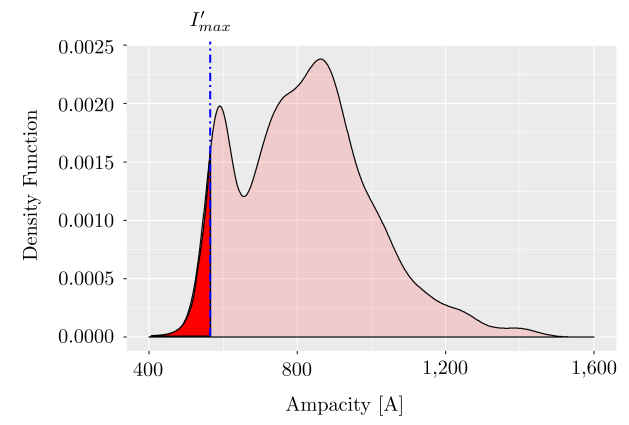

(a)

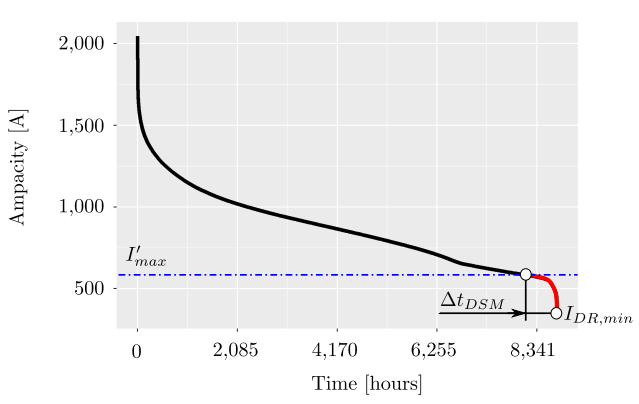

(b)

Figure 8: (a) Density plot of Ampacity; (b) Hourly distribution of DTR in the line under study.

Fig. 9 shows the time evolution of the total apparent power during one month with the simulation of the increase produced by adding the new induction reheating oven $S_{I R O}$. The values located in areas (C) and (D) in Fig. 9 produce overcurrents that have to be avoided. The points (A) and (B) show operation and maintenance operations that stop the factory.

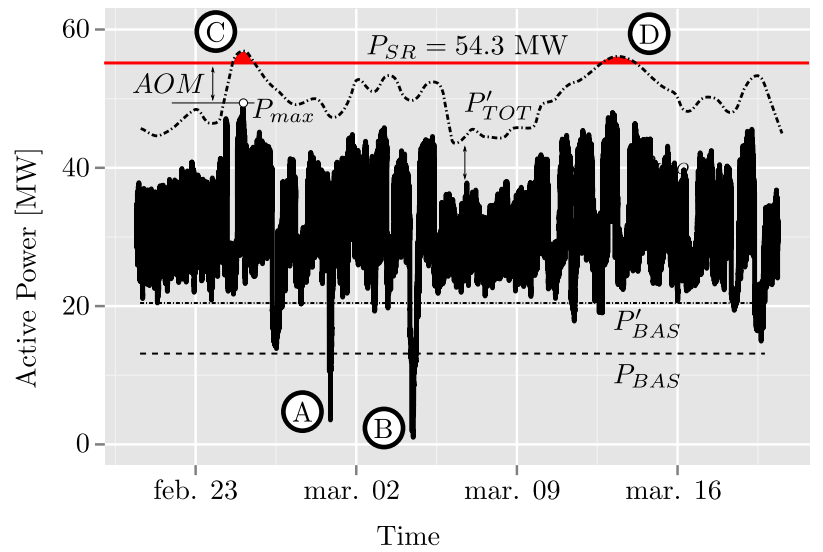

Figure 9: Simulation of the time evolution of the apparent power with the new induction reheating oven. 
Demand side management has the potential to provide important benefits to both the distribution network and the consumers. This technique is particularly useful when the loads inside the factory can be aggregated in several sets acording to their behaviour.

In the case of EAF and due to the peak-load demanded by the melting arc furnace, the DSM potential is mainly devoted to load shedding. This is due to the fact that the main aim is to minimize the electricity bill by tracking the time-varying energy price.

This paper presents a novel strategy based on the combination of demand side management and dynamic capacity management of overhead transmission lines. It has been shown that the combined strategy can be sucesfully applied to a practical problem.

The proposed methodology has been tested in a real electric arc-furnace factory, where power demand is near the static rating of the overhead transmission 315 line that supplies the energy to the industry. This factory is planning to install a new facility with an active power of $6 \mathrm{MW}$, so the total power demanded will be greater than the available capacity of the transmission line.

The simulations carried out show that the proposed methodology is able to handle the growth of the power demanded by the factory without the necessity of installing new transmission lines. From a theoretical point of view, DTR increases the capacity of existing transmission infrastructure in a significative way, even during the Summer period in which ambient temperature and wind availability reduce their cooling capacity.

The classical approach with static rating shows that the factory is not able to increase its power by adding a new facility. If $6 \mathrm{MW}$ of additional power are added to the demanded power, then the total power will be above that limit.

The application of ampacity techniques allows the factory to operate without the restriction of power demanded during 8,110 hours a year $(92.5 \%$ of the time). The results show that the proposed methodology avoids the installation 
of a new line or the repowering of the existing one.

\section{Acknowledgments}

This work was supported by the EU Regional Development Fund (FEDER) and the Spanish Government under the $\mathrm{R}+\mathrm{D}$ initiative RETOS-COLABORACION with references RTC-2015-3795-3 and RTC-2017-6782-3. The authors also ac-

knowledge Viesgo and GSW for their support.

\section{References}

[1] Kun He, Li Wang. A review of energy use and energy-efficient technologies for the iron and steel industry. Renewable and Sustainable Energy Reviews, 70:1022 - 1039, 2017.

[2] Leo S. Carlsson, Peter B. Samuelsson, Par G. Jonsson. Predicting the Electrical Energy Consumption of Electric Arc Furnaces Using Statistical Modeling. Metals, 9(9), Sep 2019.

[3] Yadollah Saboohi, Amirhossein Fathi, Igor Skrjanc, Vito Logar. Optimization of the Electric Arc Furnace Process. IEEE Transactions on Industrial Electronics, 66(10):8030-8039, Oct 2019.

[4] J. E. Runnels, M. D. Whyte, Evaluation of demand-side management, Proceedings of the IEEE 73 (10) (1985) 1489-1495. doi:10.1109/PROC.1985. 13320

[5] A. Gholian, H. Mohsenian-Rad, Y. Hua, Optimal industrial load control in smart grid, IEEE Transactions on Smart Grid 7 (5) (2016) 2305-2316. doi:10.1109/TSG.2015.2468577.

[6] M. Paulus, F. Borggrefe The potential of demand-side management in energy-intensive industries for electricity markets in germany, 2011, Applied Energy, vol. 88, no. 2, pp. $432-441$. 
[7] S. Ashok Peak-load management in steel plants, 2006, Applied Energy, vol. 83 , no. 5 , pp. $413-424$.

[8] S. S. Reka, V. Ramesh, Industrial demand side response modelling in smart grid using stochastic optimisation considering refinery process, Energy and Buildings 127 (2016) 84 - 94. doi:http://dx.doi.org/10.1016/ j.enbuild.2016.05.070.

[9] D. Ramin, S. Spinelli, A. Brusaferri, Demand-side management via optimal production scheduling in power-intensive industries: The case of metal casting process, Applied Energy, 225 (2018) 622 - 636.

[10] P. Palensky, D. Dietrich, Demand side management: Demand response, intelligent energy systems, and smart loads, IEEE Transactions on Industrial Informatics, 7 (3) (2011) 381-388. doi:10.1109/TII.2011.2158841.

[11] A. Barbato, A. Capone, Optimization models and methods for demandside management of residential users: A survey, Energies 7 (9) (2014) 5787. doi:10.3390/en7095787.

[12] K. D. McBee, J. Chong, P. Rudraraju, Demand Side Management Effects on Substation Transformer Capacity Limits, Applied Sciences, 9-16 (2019) 3266-3285. doi:10.3390/app9163266.

[13] Xiao Zhang, Gabriela Hug, and Tiro Harjunkoski. Cost-Effective Scheduling of Steel Plants With Flexible EAFs. IEEE Transactions on Smart Grid, 8(1):239-249, JAN 2017.

[14] Kristian Nolde and Manfred Morari. Electrical load tracking scheduling of a steel plant. Computers \&3 Chemical Engineering, 34(11):1899-1903, NOV 82010.

[15] Alain Hait and Christian Artigues. On electrical load tracking scheduling for a steel plant. Computers \& Chemical Engineering, 35(12):3044-3047, DEC 142011. 
[16] Pedro M. Castro, Lige Sun, and Iiro Harjunkoski. Resource-Task Network Formulations for Industrial Demand Side Management of a Steel Plant. Industrial \&3 Engineering Chemistry Research, 52(36):13046-13058, SEP 112013.

[17] I Harjunkoski and IE Grossmann. A decomposition approach for the scheduling of a steel plant production. Computers 83 Chemical Engineering, 25(11-12):1647-1660, NOV 152001.

[18] G. Dalle, J. Hernandez, I. Harjunkoski, L. Onofri, S. Engell, Demand Side Management Scheduling Formulation for a Steel Plant Considering Electrode Degradation, 12th IFAC Symposium on Dynamics and Control of Process Systems, including Biosystems DYCOPS 2019. IFAC-

(1) PapersOnLine 52 (1) (2019) 691-696. doi:10.1016/j.ifacol.2019.06. 143.

[19] K. Dehghanpour, S. Afsharnia, Designing a novel demand side regulation algorithm to participate in frequency control using iterated mappings, IET Generation, Transmission Distribution, 8 (10) (2014) 1687-1699. doi:10. 1049/iet-gtd.2013.0701.

[20] H. Goudarzi, S. Hatami, M. Pedram, Demand-side load scheduling in400 centivized by dynamic energy prices, 2011, pp. 351-356. doi:10.1109/ SmartGridComm.2011.6102346.

[21] J. S. Vardakas, N. Zorba, C. V. Verikoukis, Performance evaluation of power demand scheduling scenarios in a smart grid environment, Applied Energy 142 (2015) 164-178. doi:10.1016/j.apenergy.2014.12.060.

[22] A. Agnetis, G. De Pascale, P. Detti, A. Vicino, Load scheduling for household energy consumption optimization, IEEE Transactions on Smart Grid 4 (4) (2013) 2364-2373. doi:10.1109/TSG.2013.2254506.

[23] R. Deng, Z. Yang, J. Chen, M.-Y. Chow, Load scheduling with price uncertainty and temporally-coupled constraints in smart grids, IEEE Trans- 
actions on Power Systems 29 (6) (2014) 2823-2834. doi:10.1109/TPWRS. 2014.2311127.

[24] M. Glavan, D. Gradisar, S. Moscariello, D. Juricic, D. Vrancic, Demandside improvement of short-term load forecasting using a proactive load management - a supermarket use case. Energy \& Building, vol. 186, pg. 186-194, 2019 .

[25] IEEE Standard for calculating the current-temperature of bare overhead conductors, IEEE Std 738-2012 Draft 10 (Revision of IEEE Std 738-2006) (2012) 1-67.

[26] CIGRE TB601. Guide for thermal rating calculations of overhead lines.

[27] A. Arroyo, P. Castro, R. Martinez, M. Manana, A. Madrazo, R. Lecuna, A. Gonzalez, Comparison between ieee and cigre thermal behaviour standards and measured temperature on a $132-\mathrm{kV}$ overhead power line, Energies 8 (12) (2015) 12391. doi:10.3390/en81212391.

[28] D.P. Kroese, T. Taimre, Z.I. Botev Handbook of Monte Carlo Methods, 2011, John Wiley \& Sons

[29] H. Shabani, B. Vahidi, A probabilistic approach for optimal power cable ampacity computation by considering uncertainty of parameters and economic constraints, International Journal of Electrical Power \& Energy Systems, vol. 106, pg. 432-443, 2019. 